\title{
Hierarchical Diffusion, Aging and Multifractality
}

\author{
Hajime Yoshino* \\ Institute of Physics, University of Tsukuba, Tsukuba, Japan
}

July 26, 2021

\begin{abstract}
We study toy aging processes in hierarchically decomposed phase spaces where the equilibrium probability distributions are multifractal. We found that the an auto-correlation function, survival-return probability, shows crossover behavior from a power law $t^{-x}$ in the quasi-equilibrium regime $\left(t \ll t_{\mathrm{w}}\right)$ to another power law $t^{-\lambda}(\lambda \geq x)$ in the off-equilibrium regime $\left(t \gg t_{\mathrm{w}}\right)$ obeying a simple $t / t_{\mathrm{w}}$ scaling law. The exponents $x$ and $\lambda$ are related with the so called mass exponents which characterize the multifractality.
\end{abstract}

${ }^{*}$ Institute for Solid State Physics, Univ. of Tokyo, 7-22-1 Roppongi, Misato-ku, Tokyo, 106 Japan. (E-mail: yhajime@ginnan.issp.u-tokyo.ac.jp) 


\section{Introduction}

The aging processes, i. e. relaxational processes to approach the thermal equilibrium, are extremely slow in glassy systems like spin-glasses and one can observe remarkable aging effects in experiments[1, 2]. Among the various phenomenological descriptions of the aging effects are due to Sibani and Hoffman [3] who proposed a scenario based on the concept, hierarchical diffusion [4]. The latter concept has been implemented in many toy models [5]-[B], which we hereafter refer to as hierarchical diffusion models. The concept [10] is roughly as the following. Firstly, one considers that the free-energy landscape consists of hierarchically nested valleys, which are usually described in terms of a certain tree structure. Then one introduces a relaxational dynamics in terms of a certain master equation which describes diffusion processes between different valleys driven by thermal hoppings over the barriers. Solving the master equation, one obtains the time evolution of the distribution of probabilities to find the system at different bottoms of valleys (or leaves of the trees).

In the present paper, we consider hierarchical diffusion in a class of trees which have the following two characteristics. Firstly, we consider that the backbone structures of the trees have self-similarity as in many of the previously studied models. Secondly, we consider that the equilibrium probability distributions on the leaves are multifractal. The latter point is different from the previously studied models, whose equilibrium probability distributions are restricted to be uniform by their designs ( see however [9]). We introduce the relaxational dynamics in terms of an exactly solvable master equation.

We study aging processes after rapid temperature quenches in our model. An aging process appears as the growth of sub-trees in which the probability distributions are quasi-equilibrium (multifractal) whereas on larger scales than such sub-trees the probability distributions are still non-equilibrium (non-multifractal). As the result, a simple auto-correlation function, the survival-return probability, shows a characteristic crossover behavior: it decays by a power law $t^{-x}$ in the quasi-equilibrium regime $\left(t \ll t_{\mathrm{w}}\right)$ but by another power law $t^{-\lambda}$ in the off-equilibrium regime $\left(t \gg t_{\mathrm{w}}\right)$ and obeys a simple $t / t_{\mathrm{w}}$ scaling. The exponents $x$ and $\lambda$ turns out to be related with the

so called mass exponents which characterize the multifractal properties of the probability distributions.

The organization of this paper is the following. In section 2 , we introduce 
our hierarchical diffusion model. In section 3, we analyze the aging effect in our model focusing on the scaling properties of an autocorrelation function. In section 4 , we summarize this paper with some discussions.

\section{The Model}

\subsection{Construction of Tree}

Let us consider a system of many states which have the following clustering property. Suppose that the system can be coarse-grained so that different states merge into fewer number of states. In Fig. 1, we show such a system represented as a tree. The magnitude of the resolution power increases downward along the $h$ axis: the states (branches) are differentiated into more states (branches) as we raise the resolution power. We label the states differentiated with the maximum resolution power ( the leaves on the baselines of the trees) as $L_{i}(i=1 \ldots N)$ where $N$ is the number of such states.

For simplicity, we will only consider bifurcating trees: at every branch point we always have two branches stemming down. We will refer to the set of branches and branch points under a branch point, say $A$, as sub-tree $A$. We will refer one and the other side under a branch point $A$ as side- $I$ and $I I$ of $A$ and present them on the left and right side respectively in the figures below.

We construct the partition function of the system at the equilibrium as the following. The coarse-grained states (branches) at the same resolution level are considered to be all energetically degenerate with each other. However, the number of microscopic states they contain can vary, which means that the equilibrium local entropies and so the equilibrium statistical weights can vary as well. We consider that as a bifurcation takes place downward along the $h$ axis (the direction to increase the resolution power), the equilibrium local entropy of a coarse-grained state is partitioned into those of two substates underneath. In order words, the partition function of sub-tree $B$ is partitioned into those of side- $I$ and side- $I I$ sub-trees under $B$ with a certain partition ratio, say $1-\psi(B)$ and $\psi(B)(0 \leq \psi \leq 1)$, respectively.

Let us denote the child of $B$ (a branch point just below $B$ ) on side- $I$ as $C_{I}(B)$ and side- $I I$ as $C_{I I}(B)$. Then the ratio of the partition function of sub-tree $C_{I}(B)$ and $C_{I I}(B)$ to that of $B$, which we denote as $\pi(B, B)$, takes 
the following values,

$$
\pi(\dot{B}, B)=\left\{\begin{array}{cl}
1-\psi_{B} & \dot{B}=C_{I}(B) \\
\psi_{B} & \dot{B}=C_{I I}(B)
\end{array} .\right.
$$

It is useful to generalize the above argument as the following. Suppose that a sub-tree $D$ is enclosed by a larger sub-tree $B$. Let us denote the parent of $D$ ( the branch point just above $D$ ) as $D_{1}$ and the grand-parent of $D$ ( the parent of $D_{1}$ ) as $D_{2}$ and so on. Suppose that $B$ is the $K$ 'th ancestor of $D$, i. e. $D_{K}=B$. Then the ratio of the partition function of sub-tree $D$ to that of sub-tree $B$, which we denote as $\pi(D, B)$, can be written as the product of $\pi$ 's along the (unique) vertical path which connects $D$ and $B$,

$$
\pi(D, B) \equiv \pi\left(D, D_{1}\right) \pi\left(D_{1}, D_{2}\right) \ldots \pi\left(D_{K-1}, B\right) .
$$

For example, consider the set of leaves in a sub-tree $B$. Then $\pi\left(L_{i}, B\right)$ associated with such a leaf $L_{i}$ can be interpreted as the relative statistical weight of the leaf among the set of leaves in sub-tree $B$. We denote the highest branch point as $B_{\text {top }}$ and choose the partition function of the whole tree to be 1 . Then the equilibrium statistical weight of a leaf $L_{i}$ can be written as $\pi\left(L_{i}, B_{\text {top }}\right)$.

Here we define some other terminologies for later uses. We denote the ancestors and descendants, which are the set of branch points above and below the branch point $B$, as $\mathcal{A}(B)$ and $\mathcal{D}(B)$ respectively. We denote the set of all branch points under side- $I$ and $I I$ of $B$ as $\mathcal{D}_{I}(B)$ and $\mathcal{D}_{I I}(B)$ respectively. (Note that $\mathcal{D}_{I}(B) \cup \mathcal{D}_{I I}(B)=\mathcal{D}(B)$.) We denote the parent, grand-parent and the $K$ 'th ancestor of $B$ as $B_{1}, B_{2}$ and $B_{K}$ respectively as we already did above. We denote the lowest common ancestor of $B$ and $B$, the branch point at the top of the smallest sub-tree which enclose both $B$ and $\dot{B}$, as $A(B, \dot{B})$. For the convenience, we also introduce a hypothetical

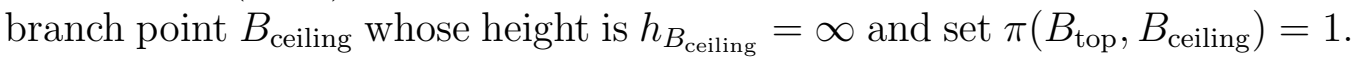

\subsection{The Master Equation}

We now introduce a stochastic dynamics of the temporal state in the hierarchically decomposed phase space. We consider that there is an thermally activated excitation associated with a branch point, say $B$, in the tree with which the temporal state can go from one to the other leaves in sub-tree $B$. 
The excitations associated with the branch points at higher $h$ are considered to have higher activation energies to be excited. Thus we now redefine the vertical axis $h$ as the scale of the activation energies of such excitations associated with the branch points.

We now introduce a simple exactly solvable dynamics which describes the stochastic jumps between the leaves, i. e. the states which are differentiable with the maximum resolution power. Here after we call the latter simply as states. Let us denote the probability to find the temporal state of the system at a state $L_{i}$ at time $t$ as $p_{i}(t)$. The time dependent distribution of the probability can be expressed in terms of a vector,

$$
\mathbf{p}(t)=\left(p_{1}(t), p_{2}(t), \ldots, p_{N}(t)\right),
$$

which should become equal to the equilibrium probability distribution in the limit $t \rightarrow \infty$. We denote the latter as as $\mathbf{p}^{\mathrm{eq}}=\left(p_{1}^{\mathrm{eq}}, p_{2}^{\mathrm{eq}}, \ldots, p_{N}^{\mathrm{eq}}\right)$ where

$$
p_{i}^{\mathrm{eq}} \equiv \pi\left(L_{i}, B_{\mathrm{top}}\right)
$$

Let us denote the transition probability to go from state $L_{j}$ to $L_{i}$ in a unit time as $\mathbf{W}_{i j}$. Then the master equation for the evolution of the probabilities can be written as

$$
\frac{d}{d t} \mathbf{p}(t)=-\boldsymbol{\Gamma} \mathbf{p}(t)
$$

with

$$
-\boldsymbol{\Gamma}_{i j}=\mathbf{W}_{i j}-\delta_{i j} \sum_{k} \mathbf{W}_{k j} .
$$

Note that the sum of the probability $\sum_{i=1}^{N} p_{i}(t)$ is always conserved.

We consider that the thermal jump process of the temporal state from a state (leaf) $L$ to another state consists of two stages. In the first stage, the excitations associated with the branch points in $\mathcal{A}(L)$ (ancestors of $L$ ) are activated in a successive manner as the following. Suppose that the excitation associated with such a branch point $B$ is activated. Then its parent $B_{1}$ get a chance to become active or not with the probability $\exp \left(-\left(h_{B_{1}}-h_{B}\right)\right)$ and $1-\exp \left(-\left(h_{B_{1}}-h_{B}\right)\right)$ respectively. If $B_{1}$ becomes active, we repeat the same trial for $B_{2}$. Otherwise, the successive ignition of the excitations stop there at the level $h_{B}$. Thus the probability that the 1 st stage ends at $B$ is

$$
\begin{aligned}
w(B \leftarrow L) & =\prod_{n=1}^{K} \exp \left(-\left(h_{A_{n}}-h_{A_{n-1}}\right)\right)\left(1-\exp \left(-\left(h_{B_{1}}-h_{B}\right)\right)\right) \\
& =\exp \left(-h_{B}\right)-\exp \left(-h_{B_{1}}\right)
\end{aligned}
$$


where $A_{0}=L$ and $A_{K}=B$.

The 2nd stage is the falling down process from the height $h_{B}$ to a leaf of sub-tree $B$. Reminding that the leaves $L \in \mathcal{D}(B)$ have different relative statistical weights $\pi(L, B)$, we expect that the probabilities to fall into the leaves depend on their amount of local entropies in such a way that those with larger amount of local entropies have more chances to receive the temporal state. So we simply choose the probability to fall down to a state (leaf) $L$ as

$$
w(\hat{L} \leftarrow B)=\pi(\dot{L}, B) .
$$

Combining the above two factors, we obtain the transition probability to go from $L$ to $L$ via $B$ as

$$
\begin{aligned}
w(\hat{L}|B| L) & =w(\hat{L} \leftarrow B) w(B \leftarrow L) \\
& =\pi(\hat{L}, B)\left[\exp \left(-h_{B}\right)-\exp \left(-h_{B_{1}}\right)\right] .
\end{aligned}
$$

Note however that such a process takes place only if both $L$ and $L$ belong to the sub-tree $B$.

The transition probability $W_{i j}$ from $L_{j}$ to $L_{i}$ is the sum of the transition probabilities over $A_{n}$ of $n=0,1, \ldots, M-1$ where $A_{0}=A\left(L_{i}, L_{j}\right)$ (the lowest

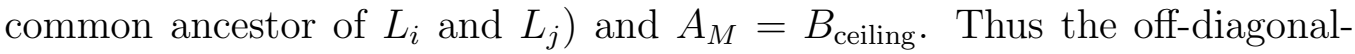
elements of the matrix $\mathbf{W}$ becomes

$$
\mathbf{W}_{i \neq j}=\sum_{n=0}^{M-1} w\left(L_{j}\left|A_{n}\right| L_{i}\right)=\sum_{n=0}^{M-1}\left[\exp \left(-h_{A_{n}}\right)-\exp \left(-h_{A_{n+1}}\right)\right] \pi\left(L_{i}, A_{n}\right),
$$

Using (41) and (10), it can be checked that the detailed balance condition

$$
\mathbf{W}_{i j} p_{j}^{\mathrm{eq}}=\mathbf{W}_{j i} p_{i}^{\mathrm{eq}}
$$

is satisfied with this choice.

\subsection{Solution of the Master Equation}

We now solve the master equation (5). The formal solution can be written as

$$
\mathbf{p}(t)=\exp (-\boldsymbol{\Gamma} t) \mathbf{p}(0)
$$


where $\mathbf{p}(0)$ is the initial distribution at $t=0$. The probability that the temporal state which initially stay at $L_{j}$ reach $L_{i}$ at time $t$ is

$$
\mathbf{G}_{i j}(t)=[\exp (-\boldsymbol{\Gamma} t)]_{i j} .
$$

In order to calculate the propagator $\mathbf{G}$, it is convenient to introduce a new matrix $\tilde{\boldsymbol{\Gamma}}$ with which we can rewrite $\boldsymbol{\Gamma}$ as

$$
\boldsymbol{\Gamma} \equiv\left(\mathbf{p}^{\mathrm{eq}}\right)^{1 / 2} \tilde{\boldsymbol{\Gamma}}\left(\mathbf{p}^{\mathrm{eq}}\right)^{-1 / 2}
$$

where $\mathbf{p}^{\mathrm{eq}}$ is the vector of equilibrium statistical weights $p_{i}^{\mathrm{eq}}$ defined in (4). Note that $\tilde{\boldsymbol{\Gamma}}$ is a real symmetric matrix so that it has real eigen values.

We now look for the $N$ eigen states of $\tilde{\Gamma}$ in a heuristic way. First of all, the static mode can be found easily as the following. The matrix $\Gamma$ satisfies $\boldsymbol{\Gamma} \mathbf{p}^{\mathrm{eq}}=0$ due to (6) and (11), from which we obtain $\tilde{\boldsymbol{\Gamma}}\left(\mathbf{p}^{\mathrm{eq}}\right)^{1 / 2}=0$. The last equation means that the vector $\left(\mathbf{p}^{\mathrm{eq}}\right)^{1 / 2}$ is an eigenvector whose eigenvalue is 0 i. e. static mode. There are $N-1$ other eigen states (dynamic modes) left to be found.

We construct here a set of vectors which consists of vectors localized under the branch points. We may call this set of vectors as umbrella set because of the localized shape of the amplitudes. On a branch point $B$ we define a vector

$$
\hat{S}_{i}(B)=\pi^{1 / 2}\left(L_{i}, B\right) u\left(L_{i}, B\right)
$$

where

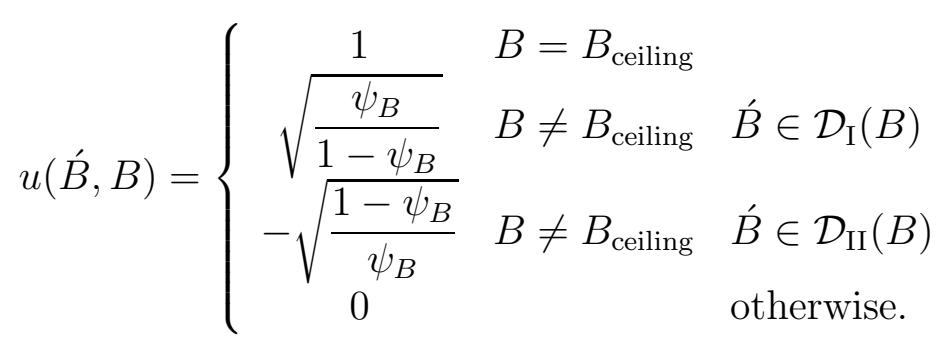

Note that the vector $\hat{S}_{i}\left(B_{\text {ceiling }}\right)$ is identical to the eigen vector of the static mode we obtained above. It can be easily checked that the vectors are normalized and orthogonal,

$$
\sum_{i} \hat{S}_{i}(B) \hat{S}_{i}(\dot{B})=\delta_{B, \dot{B}}
$$


Since there are $N-1$ eigen vectors on $N-1$ branch points and one static mode, which are all linearly independent with each other, they together constitute a complete set of dimension $N$. As shown in Appendix A, the vector $\hat{S}_{i}(B)$ actually turn out to be the correct eigen vectors of $\tilde{\boldsymbol{\Gamma}}$ which have the associated eigen values

$$
\hat{z}(B)=\exp \left(-h_{B}\right)
$$

Note that $\hat{z}\left(B_{\text {ceiling }}\right)=0$ (static mode) is endured since we have set $h_{B_{\text {ceiling }}}=$ $\infty$.

Now we rewrite some previously defined matrices in terms of the umbrella set. At first, $\tilde{\Gamma}$ becomes

$$
\tilde{\Gamma}_{i j}=\sum_{B} \hat{S}_{i}(B) \hat{z}(B) \hat{S}_{j}(B)
$$

Then using the last equation and (15) in (14), the matrix $\boldsymbol{\Gamma}_{i j}$ becomes

$$
\begin{aligned}
\boldsymbol{\Gamma}_{i j} & =\left(p_{i}^{\mathrm{eq}}\right)^{1 / 2} \tilde{\boldsymbol{\Gamma}}_{i j}\left(p_{j}^{\mathrm{eq}}\right)^{-1 / 2} \\
& =\sum_{B} \pi\left(L_{i}, B\right) u\left(L_{i}, B\right) u\left(L_{j}, B\right) \hat{z}(B) .
\end{aligned}
$$

Finally, we also rewrite the propagator $\mathbf{G}$ in terms of the umbrella set. Using (13), (14) and (19) we obtain,

$$
\begin{aligned}
\mathbf{G}_{i j}(t) & =\left(p_{i}^{\mathrm{eq}}\right)^{1 / 2} \sum_{B}\left\{\hat{S}_{i}(B) \exp (-\hat{z}(B) t) \hat{S}_{j}(B)\right\}\left(p_{j}^{\mathrm{eq}}\right)^{-1 / 2} \\
& =\sum_{B} \pi\left(L_{i}, B\right) u\left(L_{i}, B\right) u\left(L_{j}, B\right) \exp (-\hat{z}(B) t) .
\end{aligned}
$$

Using (15) and (16) and performing similar calculus shown in the appendix, we obtain the propagator in a more explicit form,

$$
\begin{aligned}
\mathbf{G}_{i j}(t) & =\sum_{n=0}^{M-1}\left[\exp \left(-\hat{z}\left(A_{n+1}\right) t\right)-\exp \left(-\hat{z}\left(A_{n}\right) t\right)\right] \pi\left(L_{i}, A_{n}\right) \\
& +\delta_{i j} \exp \left(-\hat{z}\left(A_{0}\right) t\right)
\end{aligned}
$$

where we defined $A_{0}=A\left(L_{i}, L_{j}\right)$ and $A_{1}, A_{2}, \ldots, A_{M-1}, A_{M}=B_{\text {ceiling. }}$. 


\subsection{Multifractality on Self-similar Trees}

\subsubsection{Mass Exponents}

We consider in this paper self-similar trees on which the distributions of the equilibrium statistical weights have the following multifractal characteristics. Let us define the $q$-th moments of the statistical weights [16] as,

$$
M_{q}(h) d h \equiv \overline{\sum_{\dot{B} \in \mathcal{D}(B)} \delta\left(h-\left(h_{B}-h_{\dot{B}}\right)\right) \pi^{q}(\dot{B}, B)} d h
$$

where the over-line means the average over statistically independent sub-trees $B$. Suppose that a $q$-th moment have the following scaling behavior,

$$
M_{q}(h) d h \sim \exp (\tau(q) h) d h
$$

where the exponent $\tau(q)$ is called as a mass exponent [16]. If $\tau(q)$ depends non-linearly on $q$, the distribution is regarded as multifractal.

The geometrical self-similarity appears in the 0th moment $M_{0}(h)$, which is just the number of leaves of a tree of height $h$. The mass exponent $\tau(0)$ is the fractal dimension of the tree and sometimes called as silhouette of the tree[8], which measures whether a given tree is slender or fat. A trivial exponent is $\tau(1)$ which is always 0 because of the normalization condition of the statistical weights. Another exponent which turns out to be quite important is the mass exponent of the 2 nd moment $\tau(2)$. As we see later, the two exponents $\tau(0)$ and $\tau(2)$ are related with the dynamic exponents of the survival-return probability in the present model.

\subsubsection{Randomly Branching Trees}

As an example of the trees which have the multifractal properties mentioned above, we construct here a specific class of random trees generated by the following randomly branching process (RBP). We use this specific example later when some demonstrations are necessary.

An RBP starts with a single leaf, which is regarded as the highest branch point $B_{\text {top }}$ and two branches stemming down from it. In a single step, the length of the branches under the lowest (new) branch points get longer by one unit, say $d b$ so that the height (from the baseline of the tree) of all branch points and so that the height of the tree get larger by $d b$. The branching at 
a leaf occurs with probability $p_{\text {branch }}$ in a single step. When it takes place, the leaf becomes a new branch point and two new branches starts from it. Each of such events occurs independently from each other. Repeating this procedure, we obtain a backbone structure of a tree.

Next we assign the weights on the tree. Consider a branch point $B$ and its child (the branch points just below B) $C_{I}$ on side- $I$ and $C_{I I}$ on side- $I I$. We determine the variable $\psi_{B}$ and assign $\pi\left(C_{I}, B\right)=1-\psi_{B}$ and $\pi\left(C_{I I}, B\right)=\psi_{B}$ to the branches on the side- $I$ and $I I$ of $B$. The variable $\psi_{B}$ on each branch point is chosen randomly from the distribution

$$
F(\psi) d \psi \equiv \text { probability that } \psi_{B} \text { lies between } \psi \text { and } \psi+d \psi \text {. }
$$

We perform this procedure for the whole branch points.

Consider a tree which grows larger by the RBP. A natural consequence of the RBP is that the backbone structure possesses statistical self-similarities. On the other hand, the statistical weights on the leaves are successively partitioned further into more and more fine pieces by the RBP. It is well known that if such a process is repeated, one oftenly finds very peculiar distribution of the weights : some set of pieces which have rather larger weights but negligibly smaller population compared with the typical ones come to rapidly dominate the total sum of the statistical weights (the partition function of the whole tree) as the branching proceed further. This phenomena is called as curdling [15] of multifractal objects.

As we show in Appendix B, we actually obtain the scaling property of the form (24) in the case of the random trees generated by the RBP. The mass exponent is,

$$
\tau(q)=d b^{-1} \log \left[p_{\text {branch }} \int_{0}^{1} d \psi F(\psi)\left\{\psi^{q}+(1-\psi)^{q}\right\}+\left(1-p_{\text {branch }}\right)\right] .
$$

It can be seen that it is generally non-linear with $q$. In Fig. 2 we show an example of $\tau(q)$ on random trees generated by a RBP. A special case when $\tau(q)$ becomes linear with $q$ is when the following two conditions hold: $p_{\text {branch }}=1$ (deterministic branching) and $F(\psi)=\delta(\psi-1 / 2)$ (always symmetric partition).

The real samples of such random trees can be generated numerically by by the following Monte Carlo Method. In one Monte Carlo step (MCS), the height of all branch points are raised by $d b$. Simultaneously a pseudo 
random number is generated for every leaf and if it is smaller than $p_{\text {branch }}$, a bifurcation takes place: the leaf becomes a new branch point and two new branches start from it. Fig. 1 is actually an example of such a random tree obtained by simulating the RBP of $p_{\text {branch }}=0.10, d b=1.0$ and $F(\psi)=$ $\delta(\psi-0.2)$ for 20 MCS.

\section{Aging Effect}

\subsection{Growth of Quasi-Equilibrium Domain}

We now consider an aging process after rapid temperature quench from high temperature. For this purpose, we choose the initial configuration as,

$$
\mathbf{p}(0)=\frac{1}{N}
$$

After waiting for $t_{\mathrm{w}}$ (waiting time), the probability distribution evolves up to,

$$
\mathbf{p}\left(t_{\mathrm{w}}\right)=\mathbf{G}\left(t_{\mathrm{w}}\right) \mathbf{p}(0),
$$

which eventually become $\mathbf{p}^{\mathrm{eq}}$ as $t_{\mathrm{w}} \rightarrow \infty$. Note that the initial non-equilibrium distribution is not multifractal because it is uniform, while the final fullyequilibrated distribution is multifractal. Hence the aging process in the present context can be understood as the process to approach a multifractal distribution from a non-multifractal distribution.

In order to see how the system age, it is convenient to define

$$
r_{i}\left(t_{\mathrm{w}}\right) \equiv p_{i}\left(t_{\mathrm{w}}\right) / p_{i}^{\mathrm{eq}}=\frac{1}{N} \sum_{B} \exp \left(-\hat{z}(B) t_{\mathrm{w}}\right) \pi^{-1}\left(B, B_{\mathrm{top}}\right) \tilde{u}\left(L_{i}, B\right),
$$

where we used (21) in (28) and defined

$$
\tilde{u}\left(L_{i}, B\right)=u\left(L_{i}, B\right) \sum_{j \in \mathcal{D}(B)} u\left(L_{j}, B\right) .
$$

In Fig. 3 we show an example of $r_{i}\left(t_{\mathrm{w}}\right)$ calculated using the exact solution of the master equation solved on a real sample of random tree shown in Fig. 1. One can see that, as $t_{\mathrm{w}}$ increases, $r_{i}\left(t_{\mathrm{w}}\right)$ of different states come to join with each other successively and constitute groups, among each of which 
the values of $r_{i}\left(t_{\mathrm{w}}\right)$ are common. Note that as far as the transitions within such groups are concerned, the detailed balance (11) is fulfilled. Thus we may call such a group of states as a quasi-equilibrium domain.

In order to understand the growth mechanism of the quasi-equilibrium domain in a more formal way, let us introduce a characteristic height $h^{\text {eff }}\left(t_{\mathrm{w}}\right)$ which grows logarithmically with $t_{\mathrm{w}}$,

$$
h^{\mathrm{eff}}\left(t_{\mathrm{w}}\right) \equiv \log \left(t_{\mathrm{w}}\right) .
$$

Due to the factor $\exp \left(-\hat{z}(B) t_{\mathrm{w}}\right) \simeq \exp \left(-\exp \left(h^{\mathrm{eff}}\left(t_{\mathrm{w}}\right)-h_{B}\right)\right)$, the contributions from the branch points lower than $h^{\mathrm{eff}}\left(t_{\mathrm{w}}\right)$ in the r.h.s. of the equation (29) are negligibly small, compared with those from the branch points higher than $h^{\text {eff }}\left(t_{\mathrm{w}}\right)$. So it can be roughly approximated as

$$
r_{i}\left(t_{\mathrm{w}}\right) \simeq \sum_{h(B) \gg h^{\mathrm{eff}}\left(t_{\mathrm{w}}\right)} \exp \left(-\hat{z}(B) t_{\mathrm{w}}\right) \pi^{-1}\left(B, B_{\mathrm{top}}\right) \tilde{u}\left(L_{i}, B\right) .
$$

Consider a pair of states $L_{i}$ and $L_{j}$ whose lowest common ancestor is $A\left(L_{i}, L_{j}\right)$. Suppose that after certain waiting time $t_{\mathrm{w}}^{*}$, the characteristic height $h^{\mathrm{eff}}\left(t_{\mathrm{w}}^{*}\right)$ becomes larger than $h_{A\left(L_{i}, L_{j}\right)}$. Then from the definitions (30) and (16), all the terms that survive in the sum of (32) become the same for $L_{i}$ and $L_{j}$. Consequently $r_{i}\left(t_{\mathrm{w}}\right)=r_{j}\left(t_{\mathrm{w}}\right)$ holds forever for $t_{\mathrm{w}} \gg t_{\mathrm{w}}^{*}$.

To summarize, the aging process of the present hierarchical model is understood as the growth of aged sub-trees or quasi-equilibrium domains. Here we mean by a quasi-equilibrium domain a sub-tree under a branch point lower than the characteristic height $h^{\text {eff }}\left(t_{\mathrm{w}}\right)$, which grows logarithmically with $t_{\mathrm{w}}$. The probability distributions inside an aged sub-tree is almost the same as that of fully-equilibrated one except for a common multiplicative factor. This is one of the most important consequence of hierarchical diffusion and actually found to be the case in the relaxational dynamics of microscopic spin-glass models [11].

The probability distribution inside an aged sub-tree is multifractal and possess the same scaling behaviors as that of the fully-equilibrated one while on higher scale $h \gg h^{\text {eff }}\left(t_{\mathrm{w}}\right)$, the system is still highly non-equilibrium or young in the sense that the probability distribution is not multifractal. 


\subsection{Survival-Return Probability}

We now introduce one of the simplest auto-correlation function as a prove, that is the survival-return probability,

$$
q\left(t+t_{\mathrm{w}}, t_{\mathrm{w}}\right) \equiv \sum_{i} \mathbf{p}_{i}\left(t_{\mathrm{w}}\right) \mathbf{G}(t)_{i i}
$$

It measures the probability that the system returns to the leaf where it stayed at time $t_{\mathrm{w}}$ after additional traveling of $t$.

Using (21), the autocorrelation function can be rewritten in terms of the umbrella set as

$$
\begin{aligned}
q\left(t_{\mathrm{w}}, t+t_{\mathrm{w}}\right) & =\sum_{i} \mathbf{p}_{i}\left(t_{\mathrm{w}}\right) \mathbf{G}(t)_{i i} \\
& =\sum_{B} \exp \left(-\hat{z}_{B} t\right) \sum_{i} p_{i}\left(t_{\mathrm{w}}\right) \pi\left(L_{i}, B\right) u^{2}\left(L_{i}, B\right) \\
& =\int d z \rho_{t_{\mathrm{w}}}(z) \exp (-z t)
\end{aligned}
$$

where we defined a kernel $\rho_{t_{\mathrm{w}}}(z)$ as

$$
\rho_{t_{\mathrm{w}}}(z) \equiv \sum_{B} \delta\left(z-\hat{z}_{B}\right)\left\{\sum_{i} p_{i}\left(t_{\mathrm{w}}\right) \pi\left(L_{i}, B\right) u^{2}\left(L_{i}, B\right)\right\} .
$$

In the latter subsections, we study the scaling behaviors of the survival-return

probability in random trees generated by RBP focusing on the role played by the waiting time $t_{\mathrm{w}}$.

\subsection{Two Extreme Cases}

\subsubsection{Zero Waiting Time}

We consider at first a special case of zero-waiting time case $t_{\mathrm{w}}=0$, which means that the system is in an extremely non-equilibrium condition at $t=0$. Since we have set the initial condition as (27), we obtain

$$
\begin{aligned}
\rho_{0}(z) & =\frac{1}{N} \sum_{B} \delta(z-\hat{z}(B)) \\
& \left.=\frac{1}{N} \sum_{B} \delta\left(h-h_{B}\right)\right) d h
\end{aligned}
$$


where we used (18) and defined a variable $h \equiv-\log (z)$.

Using (23) and (24), we obtain

$$
\begin{aligned}
\frac{1}{N} \sum_{B} \delta\left(h-h_{B}\right) d h & =\frac{1}{N} \sum_{B \in \mathcal{D}\left(B_{\mathrm{top}}\right)} \delta\left(\left(h_{B_{\mathrm{top}}}-h\right)-\left(h_{B_{\mathrm{top}}}-h_{B}\right)\right) \pi^{0}\left(B, B_{\mathrm{top}}\right) d h \\
& \simeq \frac{1}{N} M_{0}\left(h_{B_{\mathrm{top}}}-h\right) d h \simeq \exp (-\tau(0) h) d h \\
& \simeq z^{s-1} d z,
\end{aligned}
$$

where $s=\tau(0)$ is the silhouette. In the last equation we used $N \simeq M_{0}\left(h_{B_{\mathrm{top}}}\right)$ where $B_{\text {top }}$ is the highest branch point. In the above equations, we approximated the sums by their mean values (23) assuming that the contributions of the deviations from this mean value vanish in the thermodynamics limit $N \rightarrow \infty$, i.e. self-averaging. This assumption is valid on trees generated by the RBP because quantities on sub-trees under different branch points at the same height are statistically independent from each other.

Then using (37), we obtain a power law decay in the off-equilibrium limit

$$
q(0, t)=\int d z \Omega(z) \exp (-z t) \sim t^{-\lambda},
$$

where the exponent $\lambda$ is equal to the fractal dimension or silhouette of the tree,

$$
\lambda=s=\tau(0)
$$

The above result is similar to those of the previously studied hierarchical diffusion models, which also yield power law decays whose exponents are related with the silhouette of the trees [5]- [8]. It is, however, not surprising because the distributions of the equilibrium probabilities in such models are uniform which is also the case for the present choice of the initial condition (27).

\subsubsection{Infinite Waiting Time}

The special case of infinite waiting time $t_{\mathrm{w}}=\infty$ is also of interest. In this limit, the system is fully-equilibrated or aged at $t=0$. Using $p_{i}(\infty)=p_{i}^{\text {eq }}$ and (4), we obtain

$$
\rho_{\infty}(z)=\sum_{B} \delta(z-\hat{z}(B))\left\{\sum_{i} p_{i}^{\mathrm{eq}} \pi\left(L_{i}, B\right) u^{2}\left(L_{i}, B\right) .\right\}
$$




$$
\begin{aligned}
& =\sum_{B} \delta(z-\hat{z}(B)) \pi\left(B, B_{\mathrm{top}}\right) \sum_{i} \pi^{2}\left(L_{i}, B\right) u^{2}\left(L_{i}, B\right) \\
& =\sum_{B} \delta(z-\hat{z}(B)) \pi\left(B, B_{\mathrm{top}}\right) \Phi_{B}(z)
\end{aligned}
$$

where we defined

$$
\begin{aligned}
\Phi_{B}(z) & \equiv \delta\left(z-\hat{z}_{B}\right) \sum_{L_{i} \in \mathcal{D}(B)} \pi^{2}\left(L_{i}, B\right) u^{2}\left(L_{i}, B\right) \\
& =\frac{\psi_{B}}{1-\psi_{B}} \delta\left(z-\hat{z}_{B}\right) \sum_{L_{i} \in \mathcal{D}_{I}(B)} \pi^{2}\left(L_{i}, B\right) \\
& +\frac{1-\psi_{B}}{\psi_{B}} \delta\left(z-\hat{z}_{B}\right) \sum_{L_{i} \in \mathcal{D}_{I I}(B)} \pi^{2}\left(L_{i}, B\right) .
\end{aligned}
$$

It is sufficient to consider the scaling property of the 1st term in the last equation. We can rewrite it as,

$$
\delta\left(z-\hat{z}_{B}\right) \sum_{L_{i} \in \mathcal{D}_{I}(B)} \pi^{2}\left(L_{i}, B\right) \sim M_{2}(h) \sim z^{-\tau(2)}
$$

where we wrote $h \equiv-\log (z)$. In the first equation, we evaluated the sum by the mean value (23) assuming self-averaging property and used (24). Thus $\Phi_{B}(z)$ scales with $z$ as $\Phi_{B}(z) \sim z^{-\tau(2)}$. In the same way we obtain,

$$
\begin{aligned}
\sum_{B} \delta\left(z-\hat{z}_{B}\right) \pi\left(B, B_{\mathrm{top}}\right) d z & \sim M_{1}\left(h_{B_{\mathrm{top}}}-h\right) d h \\
& \sim \operatorname{const} \frac{d z}{z}
\end{aligned}
$$

where we wrote again $h=-\log (z)$ and used $\tau(1)=0$. Combining above results we obtain

$$
\rho_{\infty}(z) d z \sim z^{-\tau(2)-1} d z .
$$

Using (46) in (34), we finally obtain another power law in the fully-equilibrated limit,

$$
q(t+\infty, \infty) \sim t^{-x},
$$

where $x$ is an exponent defined as,

$$
x=-\tau(2) .
$$


Let us make some comments on the difference of the two dynamical exponents $\lambda$ and $x$. Generally, the inequality $\lambda \geq x$ and equivalently $\tau(0) \geq-\tau(2)$ hold as long as $\tau(q)$ decreases monotonically with increasing $q$ and concave downward. (Note that $\tau(1)=0$ holds always as we mentioned before.) The latter conditions seem to be usually satisfied. This is certainly the case on the mass exponents of trees generated by the RBP whose formula is given in (26). The equality holds only when $p_{\text {branch }}=1.0$ (deterministic branching) and $F(\psi)=\delta(\psi-0.5)$ (always symmetric partition) so that the equilibrium probability distribution becomes the same as the initial non-equilibrium (non-multifractal) distribution.

\subsection{Crossover Behavior : Aging Effect}

Now we consider the case of finite waiting time, in which we expect some waiting time effects, i. e crossover from quasi-equilibrium to off-equilibrium behavior. It is now convenient to introduce another kernel $\tilde{\rho}(z, \dot{z})$ such that

$$
\rho_{t_{\mathrm{w}}}(z) \equiv \int d \dot{z} \tilde{\rho}(z, \dot{z}) \exp \left(-\dot{z} t_{\mathrm{w}}\right)
$$

Then the autocorrelation function (34) can be rewritten as

$$
q\left(t+t_{\mathrm{w}}, t_{\mathrm{w}}\right)=\int d z \int d \dot{z} \tilde{\rho}(z, \dot{z}) \exp (-z t) \exp \left(-z t_{\mathrm{w}}\right) .
$$

From (35), (28) and (21) we obtain the explicit form of the kernel $\tilde{\rho}(z, \dot{z})$ as

$$
\begin{aligned}
\tilde{\rho}(z, \dot{z}) & =\frac{1}{N} \sum_{B} \sum_{\dot{B}} \delta(z-\hat{z}(B)) \delta(\dot{z}-\hat{z}(\dot{B})) \\
& \times\left\{\sum_{i} \pi\left(L_{i}, B\right) \pi\left(L_{i}, \dot{B}\right) u^{2}\left(L_{i}, B\right) u\left(L_{i}, \dot{B}\right) \sum_{j} u\left(L_{i}, \dot{B}\right)\right\} .
\end{aligned}
$$

The scaling form of $\tilde{\rho}(z, \dot{z})$ is studied in Appendix C. Here we read the result,

$$
\tilde{\rho}(z, \dot{z}) d z d \dot{z} \sim\left\{\begin{aligned}
z^{-\tau(2)} \frac{d z}{z} \frac{d \dot{z}}{\dot{z}} & (z>\dot{z}) \\
\left(\frac{z}{\dot{z}}\right)^{\tau(0)} \dot{z}^{-\tau(2)} \frac{d z}{z} \frac{d \dot{z}}{\dot{z}} & (z<\dot{z}) .
\end{aligned}\right.
$$


Using (52) in (50), we finally obtain

$$
q\left(t+t_{\mathrm{w}}, t_{\mathrm{w}}\right) \sim t^{\tau(2)} \tilde{q}_{1}\left(t / t_{\mathrm{w}}\right)+t^{-\tau(0)} t_{\mathrm{w}}^{\tau(0)+\tau(2)} \tilde{q}_{2}\left(t / t_{\mathrm{w}}\right),
$$

where we defined

$$
\begin{aligned}
& \tilde{q}_{1}(s) \equiv C_{1} \int d y y^{-\tau(2)-1} \exp (-y) \int^{y / s} d y^{\prime} y^{-1} \exp \left(-y^{\prime}\right) \\
& \tilde{q}_{2}(s) \equiv C_{2} \int d y y^{\tau(0)-1} \exp (-y) \int_{y / s} d y^{\prime} \dot{y}^{-(\tau(0)+\tau(2))-1} \exp \left(-y^{\prime}\right),
\end{aligned}
$$

where $C_{1}$ and $C_{2}$ are numerical prefactors.

From the above results, we find that the autocorrelation function obeys the following simple $t / t_{\mathrm{w}}$ type scaling,

$$
q\left(t+t_{\mathrm{w}}, t_{\mathrm{w}}\right) \sim t^{-x} \tilde{q}\left(t / t_{\mathrm{w}}\right),
$$

where the scaling function $\tilde{q}(y)$ behaves as

$$
\tilde{q}(y) \sim \begin{cases}\text { const } & (y \ll 1) \\ y^{x-\lambda} & (y \gg 1)\end{cases}
$$

with $x=-\tau(2)$ and $\lambda=\tau(0)$.

From the above scaling from, it can be seen that the autocorrelation function crossovers from quasi-equilibrium behavior $t^{-x}$ to off-equilibrium behavior $t^{-\lambda}$ at around $t \sim t_{\mathrm{w}}$. This crossover behavior appears due to the growth of the quasi-equilibrium domain in which the probability distribution is multifractal while on larger scale, it is still non-equilibrium (non-multifractal). The inequality of the two exponents $\lambda \geq x$ means that the off-equilibrium decay is faster than the quasi-equilibrium decay. which is intuitively satisfactory.

We show in Fig. 4 some examples of the crossover behavior of the autocorrelation function, which was obtained using the exact solutions of the master equation on real samples of random trees. The random trees are generated by Monte Carlo method which simulate the RBP of a) $p_{\text {branch }}=0.10, d b=1.0$ and $F(\psi)=\delta(\psi-0.2)$ and b) $p_{\text {branch }}=0.10, d b=1.0$ and $F(\psi)=\delta(\psi-0.5)$. The random average was took over $10^{3}$ different realizations of such trees generated by 50 Monte Carlo steps. The predicted power law $t^{-\lambda}$ and $t^{-x}$ with a) $\lambda=\tau(0)=0.0953 \ldots$ and $x=-\tau(2)=0.0325 \ldots$ (see Fig. 2) and b) 
$\lambda=\tau(0)=0.0953 \ldots$ and $x=-\tau(2)=0.0513 \ldots$ which are obtained from (26), are also included in the figure. The curvatures of the curves at lower values of $q$ are due to the finite size effects. In Fig. 5 we show the scaling plot of the data shown in Fig. 4 a). The curves of different $t_{\mathrm{w}}$ are plotted against $t / t_{\mathrm{w}}$ and shifted vertically so as to converge to a master curve. One can well see that the data are consistent with the predicted scaling laws (55) and (56).

\section{Discussions}

We have studied aging effects in a simple exactly solvable model of hierarchical diffusion. We considered the case that equilibrium probability distribution has multifractality. A specific way to generate such trees by randomly branching processes (RBP) are introduced for demonstrations. Aging processes after temperature quenches appear as the growth of aged sub-trees in which the probability distribution is in quasi-equilibrium and multifractal. In the thermodynamics limit, the height of the tree becomes infinite and the true equilibrium cannot be attained in any large but finite waiting time $t_{\mathrm{w}}$. Consequently, the waiting time dependence persists for the whole range of $t_{\mathrm{w}}$ except for $t_{\mathrm{w}}=\infty$, i. e the ergodicity is weakly broken [12]. We found that these properties clearly reflect in the survival-return probability and brings about the characteristic crossover from quasi-equilibrium behavior to off-equilibrium behavior.

Let us make some comments on the robustness of the scaling properties of the autocorrelation function we obtained in our exactly solvable model. Note that there are other possible choices of the transition matrix other than our present choice, which describe hierarchical diffusions and endures the detailed balance condition (111). For instance, one may define another transition matrix by replacing $\pi\left(L_{i}, A_{n}\right)$ in (10) by $\pi\left(L_{j}, A_{n}\right)^{-1}$. One can also construct transition matrices considering that transitions between a pair of leaves occur only over their lowest common ancestor. We investigated the solutions of the master equations with these alternative transition matrices on random trees generated by the RBP by numerical diagonalizing the transition matricies. Interestingly enough, we found that the scaling behaviors of the autocorrelation function appears essentially the same as that of the exactly solvable one presented in this paper and one only needs some renormalization 
of the global unit of time. This fact implies that the scaling properties are robust to some extent against minor changes of the model.

It is interesting to note that the crossover behavior from quasi-equilibrium behavior $\left(t \ll t_{\mathrm{w}}\right)$ to off-equilibrium behavior $\left(t \gg t_{\mathrm{w}}\right)$ obtained in the preset toy model, is very similar to that observed in the relaxational dynamics of some micro-scopic models of random systems such as 3D spin-glass model 13 and $1+1$ dimensional directed polymer in random media [14]. In the latter models, the autocorrelation functions obey $t / t_{\mathrm{w}}$ scaling law with two power law decays $t^{-x}$ at the quasi-equilibrium regime and $t^{-\lambda}$ at the off-equilibrium regime, which is what we found in the present phenomenological toy model. Thus it is tempting to speculate that our present toy model will provide a clue to understand the link between phenomenological pictures based on hierarchical diffusion and the glassy dynamics of realistic systems [18.

Acknowledgment: The author would like to thank sincerely Prof. Takayama for valuable discussions and critical reading of the manuscript. The communications with Prof. Bouchaud and Prof. Sibani are gratefully acknowledged. He would also like to thank Prof. Nemoto, Prof. Arimitsu and Dr. Hukusima for stimulating discussions and valuable comments. This work was supported by Grand-in-Aid for Scientific Research from the Ministry of Education, Science and Culture, Japan. The author was supported by Fellowships of the Japan Society for the Promotion of Science for Japanese Junior Scientists. 


\section{A The Umbrella Set}

In this appendix we show that the umbrella set defined in (15), (16) and (18) are the true eigen states of the matrix $\tilde{\Gamma}$ defined in (14). We prove this by checking if the umbrella set correctly reproduce the original transition matrix defined in (10).

Using (15) and (18) in (20) we obtain

$$
\begin{aligned}
-\Gamma_{i \neq j}= & -\sum_{B} \pi\left(L_{i}, B\right) u\left(L_{i}, B\right) \hat{z}(B) u\left(L_{j}, B\right) \\
= & \pi\left(L_{i}, A\left(L_{i}, L_{j}\right)\right) \exp \left(-h_{A\left(L_{i}, L_{j}\right)}\right) \\
& -\sum_{B \in \mathcal{A}\left(A\left(L_{i}, L_{j}\right)\right)} \pi\left(L_{i}, B\right) \exp \left(-h_{B}\right) u^{2}\left(L_{j}, B\right) \\
= & \pi\left(L_{i}, A_{0}\right) \exp \left(-h_{A_{0}}\right)-\sum_{n=1}^{M} \pi\left(L_{i}, A_{n}\right) \exp \left(-h_{A_{n}}\right) u^{2}\left(L_{j}, A_{n}\right)
\end{aligned}
$$

where we defined $A_{0}=A\left(L_{i}, L_{j}\right)$ and $A_{1}, A_{2}, \ldots, A_{M-2}, A_{M-1}=B_{\text {top }}$ and $A_{M}=B_{\text {ceiling. }}$.

We further rewrite the right hand side of the last equation as the following. The factor $u\left(L_{j}, A_{n}\right)$ in the last equation can be replaced by $u\left(A_{n-1}, A_{n}\right)$ due to the definition (16). And the factor $\pi\left(L_{i}, A_{n}\right)$ can be decomposed as $\pi\left(L_{i}, A_{n-1}\right) \pi\left(A_{n-1}, A_{n}\right)$. Then we can use the identity $\pi\left(A_{n-1}, A_{n}\right) u^{2}\left(A_{n-1}, A_{n}\right)=$ $1-\pi\left(A_{n-1}, A_{n}\right)$, which follows from (16) and (四). Then we obtain

$$
\begin{aligned}
\text { r. h. s. }= & \pi\left(L_{i}, A_{0}\right) \exp \left(-h_{A_{0}}\right) \\
& -\sum_{n=1}^{M} \pi\left(L_{i}, A_{n-1}\right)\left(1-\pi\left(A_{n-1}, A_{n}\right)\right) \exp \left(-h_{A_{n}}\right) \\
= & \sum_{n=0}^{M-1}\left[\exp \left(-h_{A_{n}}\right)-\exp \left(-h_{A_{n+1}}\right)\right] \pi\left(L_{i}, A_{n}\right)
\end{aligned}
$$

where we used $h_{A_{M}}=h_{B_{\text {ceiling }}}=\infty$ in the last equation. Then using the relation (6), we see that the off-diagonal elements of the transition probability $\mathbf{W}_{i \neq j}$ defined in equation (10) is correctly reproduced by the umbrella set.

Next we check if the matrix $\boldsymbol{\Gamma}$ written in terms of the umbrella set, properly conserve the total probability. Taking the sum over $i$ of both sides of (20) we obtain

$$
\sum_{i} \boldsymbol{\Gamma}_{i j}=\sum_{B}\left(\sum_{i} \pi\left(L_{i}, B\right) u\left(L_{i}, B\right)\right) \hat{z}(B) u\left(L_{j}, B\right)
$$


The last equation follows from the following. For $B=B_{\text {ceiling, the contri- }}$ bution is 0 since $\hat{z}\left(B_{\text {ceiling }}\right)=0$. And for $B \neq B_{\text {ceiling, }}$, one finds again zero contributions using (15),

$$
\begin{aligned}
\sum_{i} \pi\left(L_{i}, B\right) u\left(L_{i}, B\right) & =\sqrt{\frac{\psi_{B}}{1-\psi_{B}}} \sum_{L_{i} \in \mathcal{D}_{I}(B)} \pi\left(L_{i}, B\right)-\sqrt{\frac{1-\psi_{B}}{\psi_{B}}} \sum_{L_{i} \in \mathcal{D}_{I I}(B)} \pi\left(L_{i}, B\right) \\
& =\sqrt{\frac{\psi_{B}}{1-\psi_{B}}}\left(1-\psi_{B}\right)-\sqrt{\frac{1-\psi_{B}}{\psi_{B}}} \psi_{B}=0 .
\end{aligned}
$$

Thus we obtain the last equation of (58).

\section{B Mass Exponents on Random Trees}

In this appendix we study the scaling property of the $q$-th moment of the probability distribution on the random trees generated by the randomly branching process (RBP) and derive the formula (26).

Let us denote the probability that the q-th moment $M_{q}(h)$ of a random tree of size $h=m d b$ takes value $x$ as $\omega_{q}(m, x)$. Considering that larger trees can be constructed by smaller sub-trees, we obtain the following recursion relation for $\omega_{q}(m, x)$,

$$
\begin{aligned}
\omega_{q}(m+1, x) & =p_{\text {branch }} \int d y_{1} d y_{2} \int_{0}^{1} d \psi F(\psi) \omega_{q}\left(m, y_{1}\right) \omega_{q}\left(m, y_{2}\right) \\
& \times \delta\left(\psi^{q} y_{1}+(1-\psi)^{q} y_{2}-x\right)+\left(1-p_{\text {branch }}\right) \omega_{q}(m, x)
\end{aligned}
$$

In order to solve this integral equation, it is convenient to introduce a generating function defined as

$$
Z_{q}(u, m) \equiv \int d x \exp (u x) \omega_{q}(m, x)
$$

The expectation value of the q-the moment can be obtained as

$$
M_{q}(h) \simeq<x>_{q, m} \equiv \int d x x \omega_{q}(m, x)=\left.\frac{\partial}{\partial u} Z_{q}(u, m)\right|_{u=0}
$$


Multiplying $\exp (u x)$ on both sides of (59) and integrating over $x$, we obtain the recursion relation for $Z_{q}(u, M)$

$$
\begin{aligned}
Z_{q}(u, m+1) & =p_{\text {branch }} \int_{0}^{1} d \psi F(\psi) Z_{q}\left(u \psi^{q}, m\right) Z_{q}\left(u(1-\psi)^{q}, m\right) \\
& +\left(1-p_{\text {branch }}\right) Z_{q}(u, m) .
\end{aligned}
$$

Then we obtain the recursion relation for $\langle x\rangle_{q, m}$,

$$
<x>_{q, M+1}=\left[p_{\text {branch }} \int_{0}^{1} d \psi F(\psi)\left\{\psi^{q}+(1-\psi)^{q}\right\}+\left(1-p_{\text {branch }}\right)\right]<x>_{q, M} .
$$

Solving the last equation with $\langle x\rangle_{q, 1}=1$, we get

$$
M_{q}(h) \sim<x>_{q, M}=\exp (\tau(q) h),
$$

where the mass exponent $\tau(q)$ is obtained as

$$
\tau(q)=d b^{-1} \log \left[p_{\text {branch }} \int_{0}^{1} d \psi F(\psi)\left\{\psi^{q}+(1-\psi)^{q}\right\}+\left(1-p_{\text {branch }}\right)\right] .
$$

In order to further investigate the multifractal properties, it is convenient to introduce the exponent of singularity $\alpha$ defined as

$$
\pi(B, \dot{B}) \equiv \exp \left[-\alpha(B, \dot{B})\left(h_{B}-h_{\dot{B}}\right)\right]
$$

Then one can obtain the distribution of $\alpha$ or $f(\alpha)$ spectrum using the well known procedure [16] and discuss curdling. However we don't discuss about it here [17].

\section{Scaling form of Kernel}

In this appendix we study the scaling property of the kernel $\tilde{\rho}(z, \dot{z})$ with respect to $z$ and $z$. Its explicit form (51) is

$$
\begin{aligned}
\tilde{\rho}(z, \dot{z}) & =\frac{1}{N} \sum_{B} \sum_{\dot{B}} \delta(z-\hat{z}(B)) \delta(\dot{z}-\hat{z}(\dot{B})) \\
& \times\left\{\sum_{i} \pi\left(L_{i}, B\right) \pi\left(L_{i}, \dot{B}\right) u^{2}\left(L_{i}, B\right) u\left(L_{i}, \dot{B}\right) \sum_{j} u\left(L_{j}, \dot{B}\right)\right\} .
\end{aligned}
$$


Note that, from the definitions (16), the factor $u^{2}\left(L_{i}, B\right) u\left(L_{i}, \dot{B}\right)$ is non-zero only when the leaf $L_{i}$ is under both $B$ and $B$.

At first we consider the case $z>\dot{z}$. In this case, the terms which survive in the sum (67) are those in which $B$ is an ancestor of $B$ and $B$ is an ancestor of $L_{i}$. Let us introduce $h \equiv-\log (z)$ and $\dot{h} \equiv-\log (\dot{z})$. Then we obtain

$$
\begin{aligned}
& \tilde{\rho}(z, \dot{z}) d z d \dot{z} \quad(z>\dot{z})=\frac{1}{N} \sum_{\dot{B} \in \mathcal{D}\left(B_{\mathrm{top}}\right)} \delta(\dot{z}-\hat{z}(\dot{B})) \pi^{0}\left(\dot{B}, B_{\mathrm{top}}\right) \\
& \times \sum_{L j \in \mathcal{D}(\dot{B})} \delta(\dot{z}-\hat{z}(B)) \pi^{0}\left(L_{j}, \dot{B}\right) u\left(L_{j}, \dot{B}\right) \\
& \times \sum_{B \in \mathcal{D}(\dot{B})} \delta(z-\hat{z}(B)) \delta(\dot{z}-\hat{z}(\dot{B})) \pi^{1}(B, \dot{B}) u(B, \dot{B}) \\
& \times \sum_{L_{i} \in \mathcal{D}(B)} \delta(z-\hat{z}(B)) \pi^{2}\left(L_{i}, B\right) u^{2}\left(L_{i}, B\right) d z d \dot{z} \\
& \sim \frac{1}{N} M_{0}\left(h_{B_{\mathrm{top}}}-\dot{h}\right) M_{0}(\hat{h}) M_{1}(\hat{h}-h) M_{2}(h) d h d \dot{h} \\
& \sim z^{-\tau(2)} \frac{d z}{z} \frac{d \dot{z}}{\dot{z}}
\end{aligned}
$$

where we evaluated the sums by their mean values (23), assuming selfaveraging property, and used (26) and $N \simeq M_{0}\left(h_{B_{\text {top }}}\right)$.

The other case $z<z$ can be analyzed in the same way. Considering that the terms which survive in the sum (67) are those in which $B$ is an ancestor of $\dot{B}$ and $\dot{B}$ is an ancestor of $L_{i}$, we obtain

$$
\begin{aligned}
\tilde{\rho}(z, \dot{z}) d z d \dot{z} \quad(z<\dot{z}) & =\frac{1}{N} \sum_{B \in \mathcal{D}\left(B_{\mathrm{top}}\right)} \delta(z-\hat{z}(B)) \pi^{0}\left(B, B_{\mathrm{top}}\right) \\
& \times \sum_{\dot{B} \in \mathcal{D}(B)} \delta(z-\hat{z}(B)) \delta(\dot{z}-\hat{z}(\dot{B})) \pi^{1}(\dot{B}, B) u^{2}(\dot{B}, B) \\
& \times \sum_{L_{i} \in \mathcal{D}(\dot{B})} \delta(\dot{z}-\hat{z}(B)) \pi^{2}\left(L_{i}, \dot{B}\right) u\left(L_{i}, \dot{B}\right) \\
& \times \sum_{L_{j} \in \mathcal{D}(\dot{B})} \delta(z-\hat{z}(B)) \pi^{0}\left(L_{i}, \dot{B}\right) u\left(L_{i}, \dot{B}\right) d z d \dot{z} \\
& \sim \frac{1}{N} M_{0}\left(h_{B_{\mathrm{top}}}-h\right) M_{1}(h-\hat{h}) M_{2}(\hat{h}) M_{0}(\hat{h}) d h d \dot{h} \\
& \sim\left(\frac{z}{z}\right)^{\tau(0)} z^{-\tau(2)} \frac{d z}{z} \frac{d \dot{z}}{\dot{z}}
\end{aligned}
$$


where we used again $N \simeq M_{0}\left(h_{B_{\mathrm{top}}}\right)$. Combining above results, we obtain (52). 


\section{References}

[1] E. Vincent, J. Hamman, and M. Ocio, in Recent Progress in Random Magnets (World Scientific, Singapore,1992) and references there in.

[2] L. C. E Struik, 1978 Physical Aging in Amorphous Polymers and Other Materials Elsevier.

[3] P. Sibani and K. H. Hoffmann 1989, Phys. Rev. Lett. 63 2853, 1990 Z. Phys. B 80, 429.

[4] K. Hoffmann and P. Sibani 1988, Phy. Rev. A 384261.

[5] B. A. Huberman and M. Kerszberg 1985, J. Phys. A 18 L331.

[6] M. Shreckenberg 1985, Z. Phys. B 60483.

[7] A. T. Ogielski and D. L. Stein 1985, Phys. Rev. Lett. 551634.

[8] C. P. Bachas and B. A. Huberman 1987, J. Phys. A 204995.

[9] K. Nemoto, in Cooperative Dynamics in Complex Physical Systems, Hajime Takayama eds. (Springer-Verlag, 1988), has considered to mimic the dynamics of the SK model at low temperatures by a hierarchical diffusion model. He constructed random trees using information of the metastable states of the model, in which both the heights of the branch points and the statistical weights of the leaves have randomness. The present work is in part inspired by his work.

[10] R. G. Palmer 1982, Adv. In. Phys. vol. 31669 and 1987, in Heidelberg Colloquium on Glassy Dynamics J. L. van Hemmen and I. Morgenstern eds. Lecture Notes in Physics 275 (Springer-Verlag). 
[11] P. Sibani and P. Schriver 1994, Phys. Rev. B 49 6667. see also P. Sibani, J. C. Schön, P. Salamon, and J. O. Anderson 1993, Europhys. Lett. 22, 479 .

[12] J. P. Bouchaud 1992, J. Phys. (France) 21705.

[13] Heiko Rieger 1993, J. Phys. A 26, L615 and 1995 in Annual Reviews of Computational Physics II ed. D. Stauffer (World Scientific, Singapore).

[14] Hajime Yoshino 1996, J. Phys. A 29, 1421.

[15] B.B. Mandelbrot, The Fractal Geometry of Nature (Freeman, San Francisco 1982).

[16] J. Feder, Fractals (Plenum, New York, 1988)

[17] Hajime Yoshino 1996, Ph. D. thesis.

[18] In the case of SK model, which is a mean-field spin-glass model, it is knowm [20] that the participation ratio $\sum_{\alpha} W_{\alpha}^{2}$ where $W_{\alpha}$ is the equilibrium statistical weight of a pure state $\alpha$, is non-zero. The latter means that the total statistical weight is dominated by only a few pure states. Contrary, in the case of random trees generated by RBP, the participation ratio goes to zero as the height of the tree $h$ becomes infinitely high because $\tau(2)$ is negative. Thus in the case of RBP trees, the survivalreturn probability goes to zero in the limit $t \rightarrow \infty$ even after the limit $t_{\mathrm{w}} \rightarrow \infty$ is took, while it migth be non-zero in the case of SK model. I thank J. P. Bouchaud for pointing this out 19.

[19] J. P. Bouchaud, private communication. 
[20] M. Mézart, G. Parisi, N. Sourlas, G. Toulouse and M. Virasoro 1984, Phys. Rev. Lett. 521156 and J. Physique 45843. 


\section{FIGURE CAPTIONS}

Fig. 1 Hierarchical organization of states. This example is generated by a RBP explained in section 2.4.2 $\left(p_{\text {branch }}=0.10, d b=1.0, F(\psi)=\delta(\psi-0.2)\right.$, 20 MCS.) All the branches on the left side have weight $1-\psi=0.8$ while those on the right side have weight $\psi=0.2$ as indicated in the figure.

Fig. 2 The non-linear behavior of the mass exponent $\tau(q)$ v.s. $q$ : the curve is obtained using (26) for RBP of $p_{\text {branch }}=0.10, d b=1.0$ and $F(\psi)=\delta(\psi-0.2)$. The two important values of $\tau(q)$ at $q=0$ and $q=2$ are indicated by the arrows for later reference.

Fig. 3 Growth of local equilibrium domain with increasing waiting time $t_{\mathrm{w}}$ : the plot of $r_{i}\left(t_{\mathrm{w}}\right) \equiv p_{i}\left(t_{\mathrm{w}}\right) / p_{i}^{\mathrm{eq}}$ vs. $t_{\mathrm{w}}$ on the random tree shown in Fig. 2.

Fig. 4 The crossover behavior of $q\left(t_{\mathrm{w}}+t, t_{\mathrm{w}}\right)$ at different waiting times $t_{\mathrm{w}}$ on two specific kinds of random trees a) and b) (see text). The waiting time $t_{\mathrm{w}}$ varies as $t_{\mathrm{w}}=0,10,10^{2}, \ldots, 10^{14}, \infty$ from the lowest to the top curve.

Fig. 5 The $t / t_{\mathrm{w}}$ scaling plot of $q\left(t_{\mathrm{w}}+t, t_{\mathrm{w}}\right)$ : the data of of different $t_{\mathrm{w}}$ presented in Fig. 5 a) except $t_{\mathrm{w}}=0$ and $\infty$, are used in this plot. The vertical scale is arbitrary. 
FIGURES 


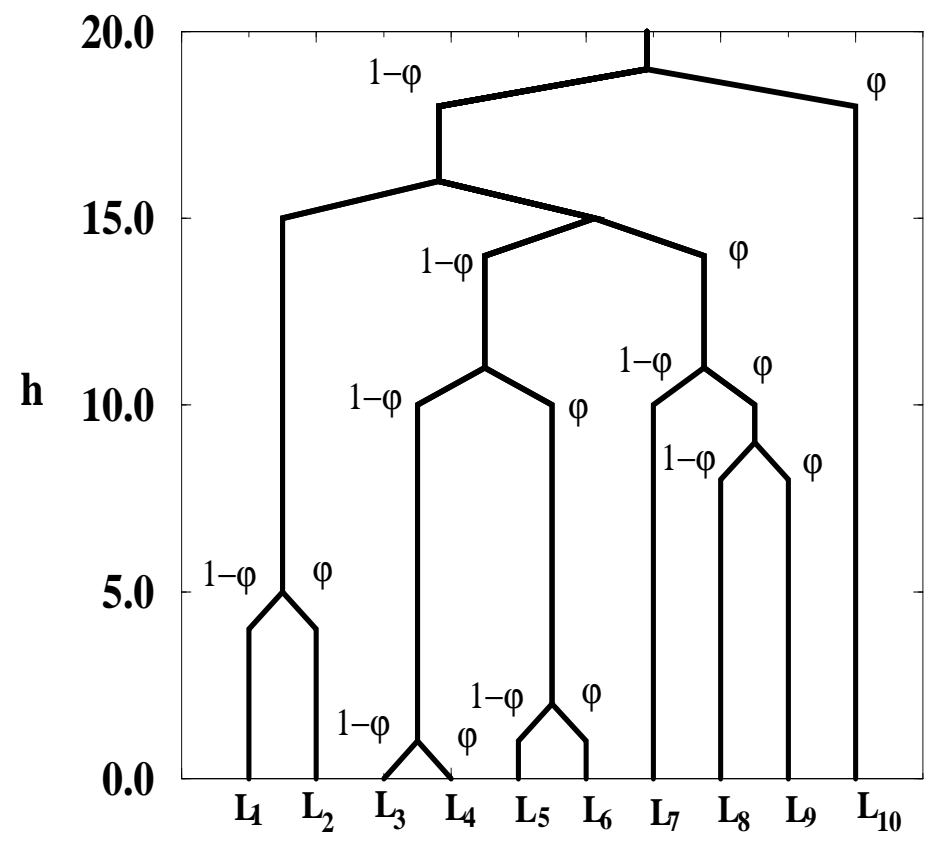

Fig. 1 


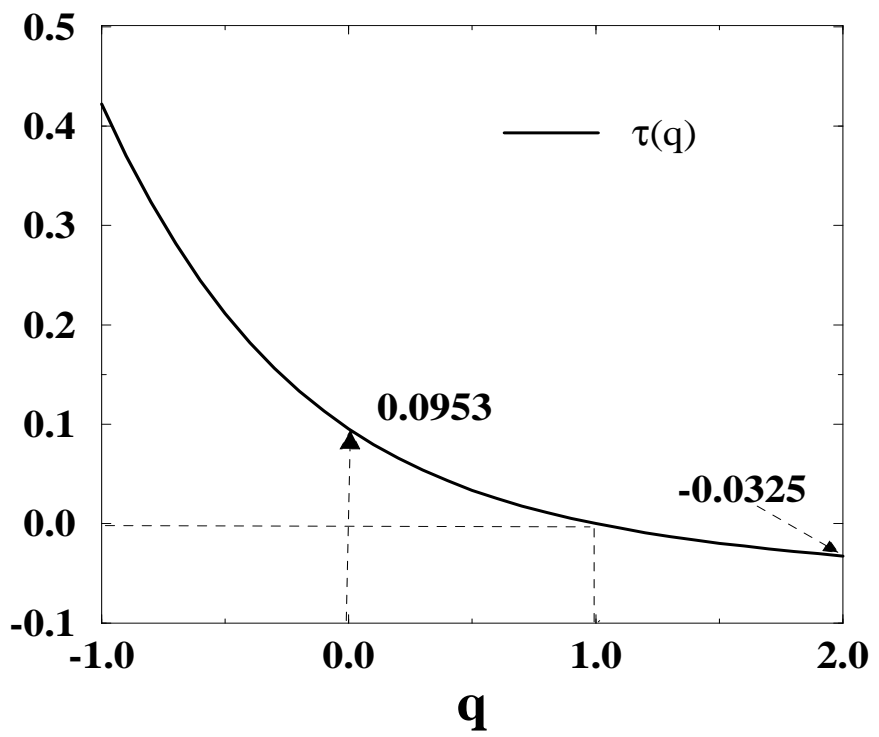

Fig. 2 


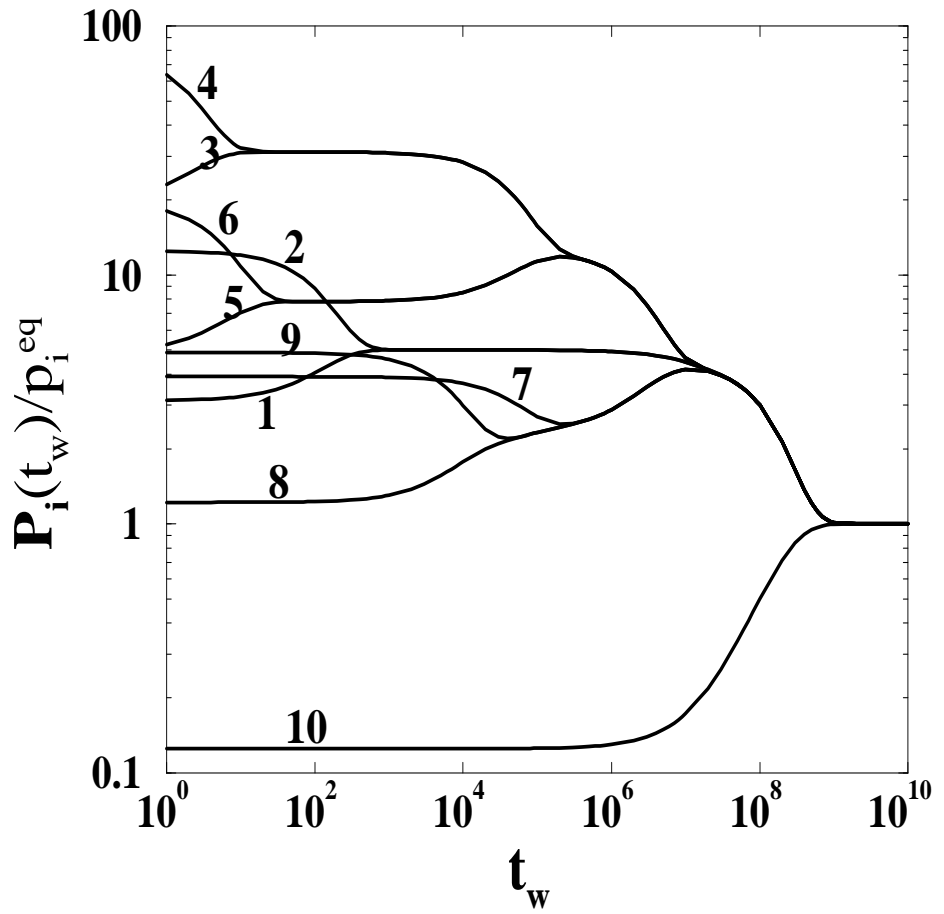

Fig. 3 

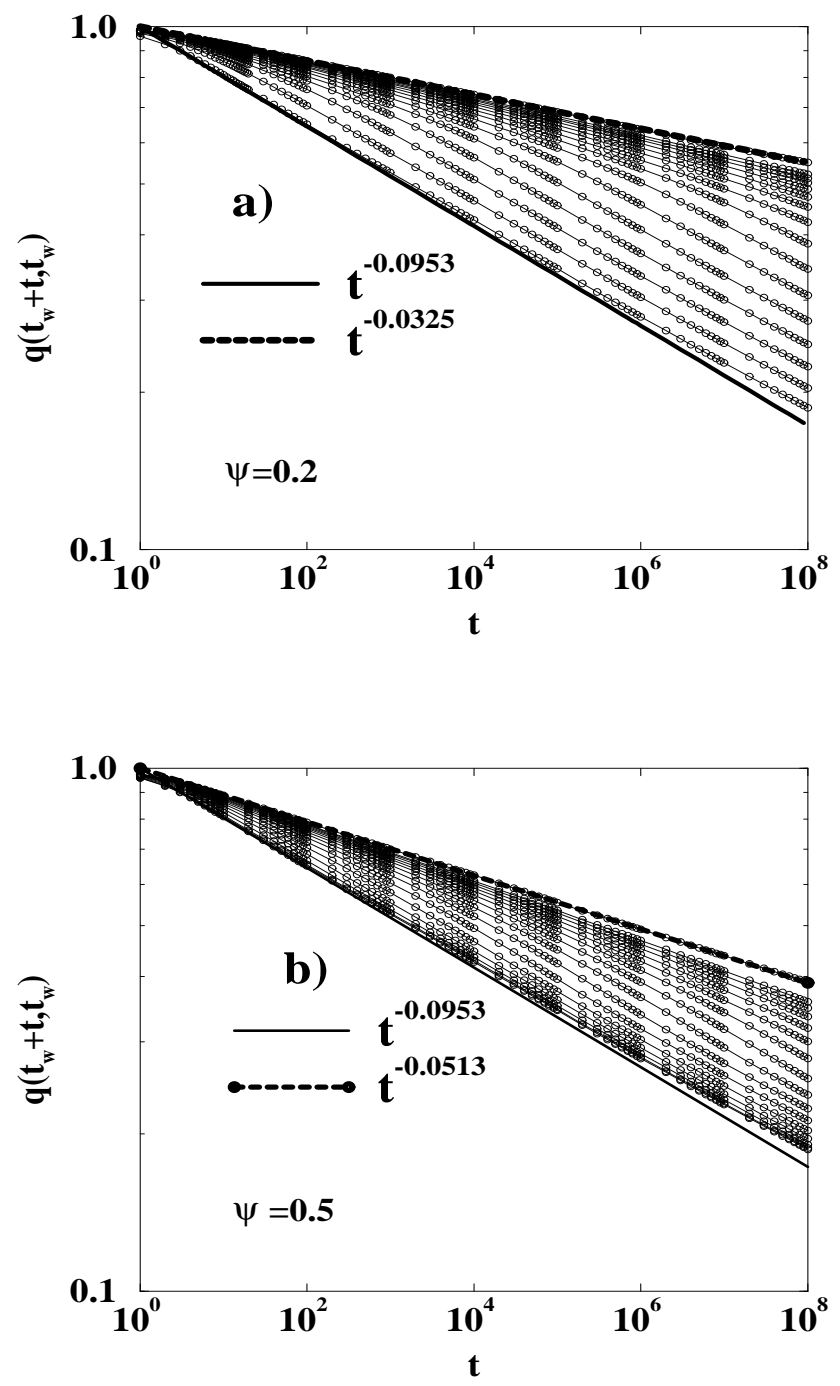

Fig. 4 


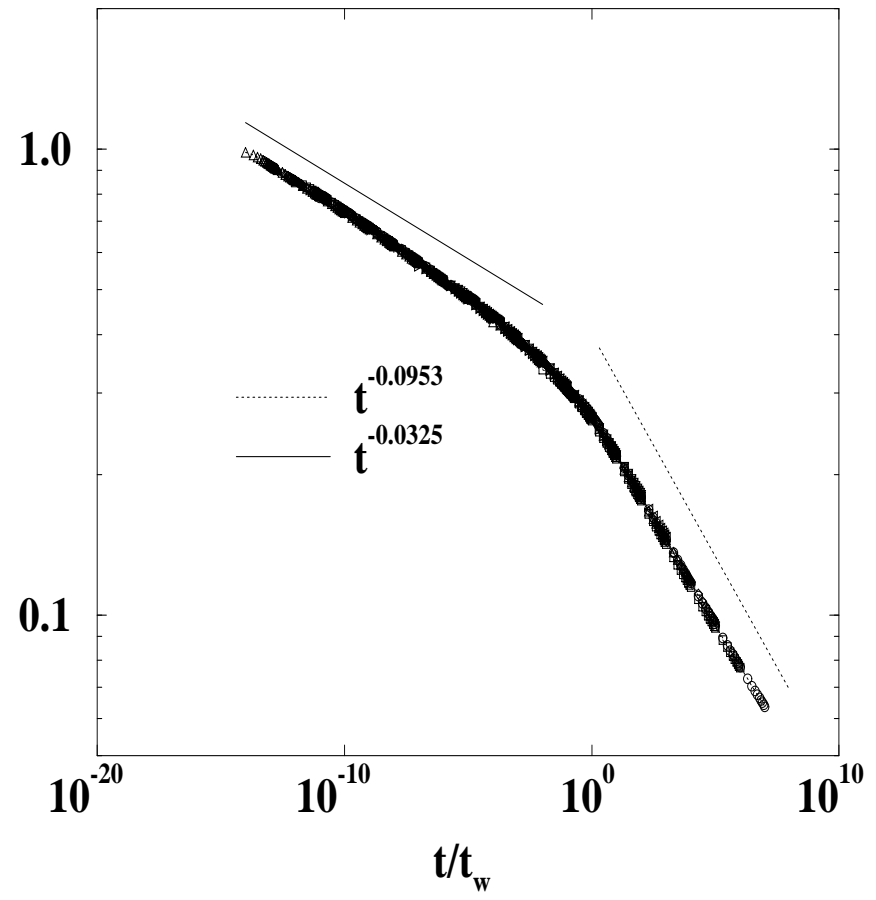

Fig. 5 


\section{LIST OF CHANGES MADE IN THIS REVISED VERSION}

- The subsection which was titled 'Distribution of Relaxation Times' in the original version, which was placed just after subsection 3.2, was removed and the necessary part of it is now included in subsection 3.3.1.

- The figure 1 of the original version, which was intended to explain the idea of hierarchical decomposition of the phase space, was removed. This is because figure 2 in the original version (figure 1 of the revised version), which shows an example of random trees generated by RBP, can be used to explain the idea.

- The list of references were updated and some additional references ( the last three of it) are added.

- Minor changes such as corrections of miss typing and refinements of some sentences are made. 